Review Article

\title{
Cerebrospinal fluid (CSF) directed antineoplastic agents: a review of current knowledge
}

\begin{abstract}
Central nervous system (CNS) neoplasms are a heterogeneous group of lesions that are classified as either primary or secondary based on their location of origin. ${ }^{1}$ Primary CNS neoplasms are tumors that arise in the brain parenchyma; these include gliomas, meningiomas, nerve sheath tumors, and pituitary tumors. ${ }^{1,2}$ Brain metastases, or secondary brain neoplasms, are tumors that arise from a primary cancer elsewhere in the body and migrate through the bloodstream or lymphatic system to the brain parenchyma, the dura, or the leptomeninges. ${ }^{2}$ Nearly any type of cancer can metastasize to the brain; however, the most common brain metastases originate from lung cancer, breast cancer, melanoma, renal cancer, or colorectal cancer, listed in decreasing order of prevalence. ${ }^{1}$ The efficacy of CSF directed antineoplastic agents for the treatment of brain neoplasms depends on multiple poorly understood factors (Table 1). ${ }^{3}$ Current treatment strategies aimed at increasing CNS antineoplastic concentrations include intrathecal (IT) therapy, radiation therapy, and highdose intravenous therapy. ${ }^{4}$ This review focused on the pharmacology of antineoplastic agents administered directly into the CSF in the current clinical setting, and how these pharmacology concepts are translated into the clinical setting.
\end{abstract}

Keywords: brain metastasis, antineoplastic agents
Volume 2 Issue I - 2015

\author{
Laura Molander, Catherine Caraway, Linda \\ Linderbeck, Damian A Laber \\ Moffitt Cancer Center, University of South Florida, USA
}

Correspondence: Damian A Laber, Division of Hematology/ Oncology, I 3220 USF Laurel Drive, MDF 4|49, Tampa, FL 336I2, USA, Tel 8I3-974-3725, Email damian.laber@gmail.com

Received: November 15, 2014 | Published: January 28, 2015
Abbreviations: CNS, central nervous system; IT, intrathecal; ISF, interstitial fluid; BBB, blood-brain barrier; DNL, disseminated necrotizing leuko-encephalopathy; IV, intravenous; FDA, food and drug administration

\section{Barriers to drug transport}

There are three proposed barrier systems to drug transport in the brain: the blood-CSF barrier, the blood-brain barrier (BBB), and the CSF-brain barrier. ${ }^{2}$ The first barrier refers to the epithelial cells of the choroid plexus that resides within the CSF of the lateral, third, and fourth ventricles..$^{2,5,6}$ This barrier regulates drug transfer from the blood to the $\mathrm{CSF}^{6}{ }^{6}$ The second barrier refers to the capillary endothelial cells

Table I Antineoplastic agents currently used for intrathecal administration

\begin{tabular}{lllll}
\hline Drug & Dose & Interval & $\begin{array}{l}\text { CSF } \\
\text { Half-life* }\end{array}$ & Common Indications \\
\hline MTX $^{10,24}$ & 8 to $12 \mathrm{mg}$ & 2 to 5 days & $\sim 9$ hours & Meningeal leukemia \\
Ara-C & I00 mg/dose & $\begin{array}{l}2 \text { to } 3 \text { times } \\
\text { weekly }\end{array}$ & 3.4 hours & Meningeal leukemia \\
$\begin{array}{l}\text { Liposomal } \\
\text { cytarabine }\end{array}$ & $\begin{array}{l}\text { Adults: } 50 \mathrm{mg} \\
\text { Peds: } 35 \mathrm{mg}\end{array}$ & Every 2 weeks & 14I hours & Refractory leptomeningeal leukemia \\
\hline
\end{tabular}

*Under normal circumstances

\section{Biology of the CSF}

Constituents of the CSF provide nourishment to and facilitate electrical activity within the CNS. ${ }^{8}$ In the normal adult, CSF is clear, colorless, and acellular. Several macromolecules, such as glucose and protein, persist naturally within the CSF. Protein concentrations range from 23 to $38 \mathrm{mg} / \mathrm{dL}$ and glucose concentrations are expressed as a blood to CSF ratio of 0.6 , with higher glucose concentrations observed within the ventricles than the lumbar space. ${ }^{7}$ Major CSF electrolytes include $\mathrm{Na}^{+}, \mathrm{K}^{+}, \mathrm{Ca}^{2+}, \mathrm{Mg}^{2+}, \mathrm{Cl}^{-}$, and bicarbonate ion. ${ }^{8}$ of the brain. This barrier regulates drug transfer from the blood to the interstitial fluid (ISF) that bathes the CNS parenchyma. ${ }^{2,6}$

The infrastructure of these first two barriers is quite different, owing to the varied permeability between them. Lipid-soluble, nonpolar molecules with a molecular mass of less than 400 to 600 Daltons are readily transported through the BBB whereas virtually all small and large molecules can traverse the blood-CSF barrier. ${ }^{6,7}$ The final barrier refers to the epithelial cells of the ependyma and pia membranes that line the ventricles and subarachnoid spaces, respectively. ${ }^{8}$ This barrier regulates drug transfer from the CSF to the CNS parenchyma through diffusion. ${ }^{2,8}$ In addition to these physical barriers, efflux pumps, drug metabolizing enzymes, and bulk flow impede effective drug delivery to the CNS parenchyma. ${ }^{2}$ 
normal adult, CSF is produced at a bulk flow rate of 0.3 to $0.4 \mathrm{~mL} / \mathrm{min}$ to maintain a volume of 90 to $150 \mathrm{~mL} .{ }^{5}$ CSF flows in a unidirectional manner via the cerebral aqueduct through the lateral, third, then fourth ventricles and finally through the subarachnoid spaces prior to being absorbed into the venous blood at the level of the arachnoid villi or drained into the cervical lymphatics. ${ }^{5,79}$ The entire CSF volume is replaced every 4 to 5 hours or 4 to 5 times each day. ${ }^{2,6}$ It is important to note that neoplastic meningitis and bulky CNS disease may obstruct the physiologic CSF flow and normal rates of CSF production and resorption (Figure 1). ${ }^{4}$

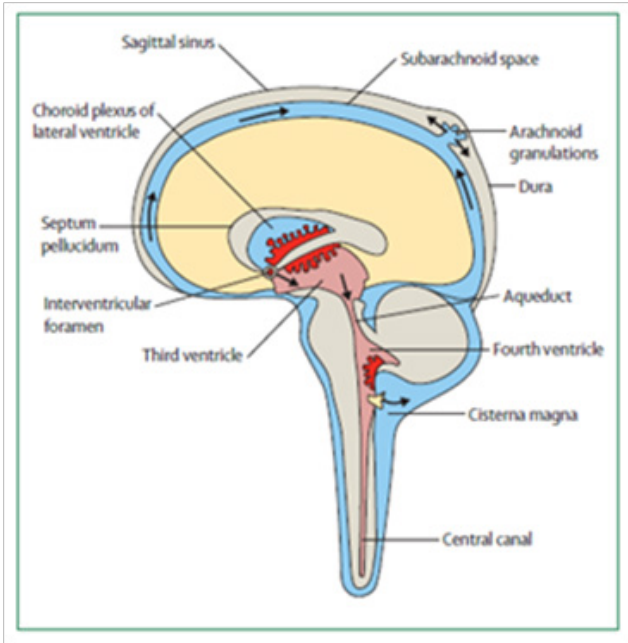

Figure I CSF flow in the CNS. ${ }^{12}$

\section{Methods of IT drug delivery}

There are two routes of IT drug delivery used in clinical practice: intralumbar and intraventricular injections. Intralumbar is administered by a lumbar puncture and intraventricular is achieved through the use of an access device, such as an Ommaya reservoir. Intralumbar might be the preferred route of entry due to its simplicity; however, an intraventricular access device facilitates serial administration of antineoplastic agents and CSF sampling. ${ }^{10}$ Drug distribution following lumbar puncture is non uniform due to the directionality of CSF circulation. As a result, increased exposure to drug metabolizing enzymes and efflux systems may compromise effective drug delivery. ${ }^{4}$ Following intraventricular injection, a drug traverses the CSF flow tracks and is rapidly absorbed into systemic circulation at the arachnoid villi. Then, the drug re-enters the CNS parenchyma through the BBB or the blood-CSF barrier. ${ }^{6}$ Drugs most commonly used for IT antineoplastic treatment include: methotrexate, Ara-C, and liposomal cytarabine.

\section{Concurrent radiotherapy}

IT antineoplastic therapy with concurrent brain radiotherapy can result in injury to the CNS at higher rates than those of single modality treatment. ${ }^{11}$ Complications of combined treatment include: tumor pseudoprogression, leukoencephalopathy, and disseminated necrotizing leukoencephalopathy (DNL). Tumor pseudoprogression mimics tumor recurrence both clinically and diagnostically..$^{12,13}$ It occurs as a result of necrosis secondary to vascular damage and subsequent ischemia with an estimated incidence of approximately 30 percent. Leukoencephalopathy is often more severe and occurs more frequently, with incidence rates ranging from 30 to 75 percent. It occurs as a result of demyelination or frank degeneration of white matter. Clinically, it presents as mild to severe cognitive impairment. ${ }^{12}$
DNL is the most severe toxicity of concurrent radiotherapy. It is essentially widespread leukoencephalopathy and presents as cognitive, motor, and autonomic deficits. ${ }^{11,12}$ The incidence rate of DNL is reported to be approximately 24 percent. Currently, there are no prophylactic or curative treatments available for CNS toxicity from combined treatment. ${ }^{12}$ The use of concurrent radiotherapy and CSF directed chemotherapy should be discouraged until safety could be improved.

\section{Drug specific information \\ Methotrexate (MTX)}

Methotrexate was first used as an antineoplastic agent in 1959 for the treatment of primary and metastatic brain tumors. ${ }^{4}$ It is a cell-cycle phase specific folate antimetabolite that inhibits DNA synthesis, repair, and cellular replication. Routes of CNS administration can include intravenous (IV) and IT, with the latter being more advantageous due to the aforementioned barriers of drug transport. ${ }^{14}$ Pharmacokinetic studies have shown negligible CSF ventricular concentrations following a single IV injection, with greater concentrations achieved following high-dose IV infusion; however, therapeutic concentrations are rarely achieved intravenously. ${ }^{10}$ IT administration circumvents the BBB; following intraventricular administration, therapeutic concentrations are maintained for a full 48 hours in both ventricular and lumbar fluids, with equilibration observed within 4 hours. ${ }^{15}$ Methotrexate exhibits biphasic elimination from the CSF with halflives of 4.5 and 14 hours for the intervals of 4 to 36 hours and 48 to 96 hours. ${ }^{16,17}$ Increased half-lives at later times can be explained by the reverse diffusion of methotrexate from the CNS parenchyma back into the CSF after bulk flow had initially diluted the CSF concentration. ${ }^{10}$ Methotrexate elimination occurs primarily by CSF bulk flow resorption. ${ }^{4}$

\section{Cytarabine (Ara-C)}

Cytarabine (Ara-C) is an antineoplastic agent approved by the Food and Drug Administration (FDA) in 1969 for prophylaxis and treatment of meningeal leukemia. It is a cell-cycle phase specific pyrimidine analog that inhibits DNA synthesis, repair, and cellular replication. ${ }^{4}$ Routes of CNS administration include IV and IT; however, IV administration allows for minimal penetration across the BBB into the CSF. When administered intravenously, Ara-C is metabolized intracellularly to its active metabolite, cytosine arabinoside triphosphate (Ara-CTP). Continuous IV infusions allow for a steady state Ara-C CSF-to-plasma ratio of $0.4 .{ }^{18}$ Plasma Ara-C exhibits biphasic elimination with half-lives of 10 minutes and 1 to 3 hours. Conversely, Ara-C elimination from the CSF exhibits firstorder kinetics, with a clearance of $0.42 \mathrm{~mL} / \mathrm{min}$ and a half-life of 3.4 hours. ${ }^{5,19}$ Following IT administration, the concentration of Ara-C is undetectable in the plasma due to rapid conversion to Ara- $U$ by cytidine deaminase. Levels of this enzyme are markedly decreased in the CSF, resulting in sustained concentrations. ${ }^{4,19,20}$ Ultimately, Ara-C is eliminated from the CSF through a collective process that includes enzymatic degradation and CSF bulk flow.

\section{Liposomal Cytarabine}

Liposomal cytarabine received FDA approval in 1999 for the treatment of lymphomatous meningitis. Historically, neoplastic meningitis was treated with continuous infusions of antineoplastic agents to obtain prolonged therapeutic concentrations in the CSF. ${ }^{21}$ Liposomal cytarabine, which is administered by lumbar puncture or Ommaya reservoir, is formulated using encapsulation technology to 
maximize the efficacy of cytarabine by prolonging CSF exposure. ${ }^{4}$ Administration of liposomal cytarabineyields peak CSF levels of cytarabine ranging from 30 to $50 \mathrm{mcg}$ within the first hour, with a variable half-life of 5.9 to 82.4 hours. ${ }^{22}$ This unique liposomal formulation extends the serum half-life of cytarabine nearly 40fold, from 3.4 hours to over 141 hours, allowing for dosing every 2 weeks as opposed to multiple weekly doses seen with standard cytarabine. ${ }^{4}$ Liposomal cytarbine is able to maintain cytotoxic levels in the CSF, both in the lumbar and ventricular fluid, for over 14 days post administration. This extended CSF circulation allows for fewer side effects due to toxicity yet maintains necessary cytotoxic levels; however, any blockage or disruption of the CSF flow can lead to elevated concentrations of free cytarabine in the CSF, resulting in a greater risk of neurotoxicity. Plasma concentrations following IT administration are negligible. Due to significantly lower levels of cytidine deaminasein the CSF, clearance of liposomal cytarabine is similar to the bulk flow rate, roughly $0.24 \mathrm{~mL} / \mathrm{min} .^{22-25}$

\section{Conclusion}

CSF directed treatment is an effective model for administration of antineoplastic agents. Understanding the pharmacokinetic concepts associated with this route of administration has allowed clinicians to optimize the safe and effective use of these agents. Since the outcome of most brain malignancies remains poor, studies of newer agents are eagerly warranted.

\section{Acknowledgments}

None.

\section{Conflicts of interest}

Authors declare that there is no conflict of interest.

\section{References}

1. Hsu SH, Alfred Y, Anita M, et al. Tumors of the Central Nervous System. In: Hagop MK et al. (Eds.), The MD Anderson Manual of Medical Oncology. 2nd ed. McGraw-Hill: New York, USA; 2011. 1400 p.

2. Fleischhack Gudrun, Ulrich Jaehde, Udo Bode. Pharmacokinetics Following Intraventricular Administration of Chemotherapy in Patients with Neoplastic Meningitis. Clin Pharmacokinet. 2005;44(1):1-31.

3. Wilson TR, Longley DB, Johnston PG. Chemoresistance in Solid Tumours. Ann Oncol. 2006;17(Suppl 10):x315-x324.

4. Berg Stacey, Susan M Blaney. Intrathecal Administration. Handbook of Anticancer Pharmacokinetics and Pharmacodynamics. 2nd ed. Springer: New York, USA; 2014. 457-476 p.

5. Brinker, Thomas, Edward Stopa, et al. A New Look at Cerebrospinal Fluid Circulation. Fluids Barriers CNS. 2014;11:10.

6. Pardrigde William M. Drug Transport in Brain via the Cerebrospinal Fluid. Fluids Barriers CNS. 2011;8(1):7.

7. Johnson Kimberly S, Daniel J Sexton. Cerebrospinal Fluid: Physiology and Utility of an Examination in Disease States. Up To Date, Wolters Kluwer Health; 2014.
8. Cohen Don. The Fluid Medium: CSF-An Introduction to Craniosacral Therapy: Anatomy, Function, and Treatment. North Atlantic, Berkeley, USA; $1995.3-20 \mathrm{p}$.

9. Veening JG, Henk PB. The Regulation of Brain States by Neuroactive substances distributed via the cerebrospinal fluid; a review. Cerebrospinal Fluid Res. 2010;7:1.

10. Collins Jerry M. Pharmacokinetics of Intraventricular Administration. $J$ Neurooncol. 1983;1(4):283-291.

11. Kim JY, Kim ST, Nam DH, et al. Leukoencephalopathy and Disseminated Necrotizing Leukoencephalopathy Following Intrathecal Methotrexate Chemotherapy and Radiation Therapy for Central Nerve System Lymphoma or Leukemia. J Korean Neurosurg Soc. 2011;50(4):304-310.

12. Soussain C, Damien R, John RF, et al. CNS Complications of Radiotherapy and Chemotherapy. Lancet. 2009;374(9701):1639-1651.

13. Brandes AA, Alicia T, Federica S, et al. Disease Progression or Pseudoprogression after Concomitant Radiochemotherapy Treatment: Pitfalls in Neuro-oncology. Neuro Oncol. 2007;10(3):361-367.

14. Gleissner B, Marc CC. Neoplastic Meningitis. Lancet Neurol. 2006;5(5):443-452.

15. Shapiro WR, Dean FY, Bipin MM. Methotrexate: Distribution in Cerebrospinal Fluid after Intravenous, Ventricular and Lumbar Injections. N Engl J Med. 1975;293(4):161-166.

16. Bleyer Werner, Robert Dedrick. Clinical Pharmacology of Intrathecal Methotrexate. I. Pharmacokinetics in Nontoxic Patients after Lumbar Injection. Cancer Treat Rep. 1977;61(4):703-708.

17. Methotrexate. Mayne Pharma Inc, New Jersey USA; 2005.

18. Fulton DS, Victoria AL, Philip HG, et al. Intrathecal Cytosine Arabinoside for the Treatment of Meningeal Metastases from Malignant Brain Tumors and Systemic Tumors. Cancer Chemother Pharmacol. 1982;8(3):285-291.

19. Zimm S, Collins JM, Miser J, et al. Cytosine Arabinoside Cerebrospinal Fluid Kinetics. Clin Pharmacol Ther. 1984;35(6):826-830.

20. Przuntek H, Breithaupt H. Cytarabine: Distribution in Ventricular Cerebrospinal Fluid after Lumbar Injection. JNeurol. 1981;226(1):73-76.

21. Kim S, Chatelut E, Kim JC, et al. Extended CSF Cytarabine Exposure following Intrathecal Administration of DTC 101. J Clin Oncol. 1993;11(11):2186-2193.

22. DepoCyt. Pacira Pharmaceuticals. San Diego, California, USA; 2011.

23. Chamberlain Marc C. Neurotoxicity of Intra-CSF Liposomal Cytarabine (DepoCyt) Administered for the Treatment of Leptomeningeal Metastases: A Retrospective Case Series. J Neurooncol. 2012;109(1):143-148.

24. Glantz MJ, Jaeckle KA, Chamberlain MC, et al. A Randomized Controlled Trial Comparing Intrathecal Sustained-release Cytarabine (DepoCyt) to Intrathecal Methotrexate in Patients with Neoplastic Meningitis from Solid Tumors. Clin Cancer Res. 1999;5(11):3394-3402.

25. Cytostar. Kirkland, Quebec, Pfizer Canada. 